\title{
Uso de Learning Analytics sobre dados de simulados para apoio à avaliação da aprendizagem por professores e gestores
}

\author{
Adelson Menezes Portela-UFAM - adelson.portela@icomp.ufam.edu.br \\ Gabriel de Souza Leitão -UFAM - gabriel.leitao@icomp.ufam.edu.br \\ Raimundo da Silva Barreto -UFAM - rbarreto@icomp.ufam.edu.br \\ Elaine Harada Teixeira de Oliveira -UFAM - elaine@icomp.ufam.edu.br
}

\begin{abstract}
Resumo. A avaliação da aprendizagem a partir de ambientes virtuais de aprendizagem (AVA) traz novas oportunidades, porém produz um grande volume de dados, o que dificulta uma análise manual dos resultados dos alunos. Por esse motivo, este trabalho busca fornecer informações relevantes para professores e gestores a partir da utilização de técnicas de Learning Analytics (LA) sobre dados de simulados aplicados em um AVA. Seguindo o processo de LA e suas técnicas, foi possivel visualizar em quais turmas os alunos apresentaram menor compreensão em relação à disciplina, agrupar as disciplinas conforme os valores das métricas de avaliação da aprendizagem utilizadas, assim como verificar que o tempo de resposta das questões, estipulado pelo professor, pode ter sido insuficiente.
\end{abstract}

Palavras-Chave: Avaliação da aprendizagem. Learning Analitycs. Ambientes Virtuais de Aprendizagem.

\section{Use of Learning Analytics on simulated data to support learning assessment by teachers and managers}

Abstract. The assessment of learning from a virtual learning environment (VLE) brings new opportunities, but produce a large volume of data, which makes it difficult to manually analyze student results. For this reason, this work seeks to provide relevant information for teachers and managers from the use of Learning Analytics (LA) techniques on simulated data applied in an AVA. Following the LA process and its techniques, it was possible to understand which classes have less comprehension in almost all the subjects analyzed, group the subjects according to the values of the learning assessment metrics used, as well as verify that the response time to the questions, stipulated by the teacher, was underestimated.

Keywords: Learning Assessment. Learning Analytics. Virtual Learning Environments.

\section{Introdução}

Enquanto em sala de aula presencial, os professores coletam informações pela observação dos alunos e através das notas obtidas nas avaliações, nos Ambientes Virtuais de Aprendizagem (AVA) essas informações estão disponíveis a partir dos dados de interação dos alunos com o ambiente virtual. Entretanto, o volume de dados gerados, torna inviável análises manuais dos $\operatorname{logs}$ de interação dos alunos, o que de acordo com Martic (2017) pode ser solucionado com o uso de Learning Analytics (LA).

Porém, como afirmam Paiva, Bittencourt e Lemos (2019), treinar os professores de diferentes áreas de conhecimento para utilizar essa técnica demanda tempo e esforço, sendo que a eficácia não é garantida. Isso é devido a Learning Analytics não ser uma 
área de pesquisa isolada, mas estar relacionada a diferentes campos de pesquisa, como a Mineração de Dados Educacionais (MDE), sistemas de recomendação, Web Analytics, Business Intelligence e sistemas de aprendizagem adaptativa, dentre outras (Chatti et al., 2012; Pineda e Cadavid, 2018).

Conforme Siemens (2010), Learning Analytics é "a medição, coleta, análise e relatório dos dados dos alunos e seus contextos, com o objetivo de entender e otimizar a aprendizagem e o ambiente em que ela ocorre". Nesse contexto, Chatti et al. (2012) apresentam um modelo de processo de Learning Analytics como um ciclo, que segue 3 fases distintas: Coleta e Pré-Processamento, Análise e Ação e Pós-Processamento.

Nesse aspecto, este trabalho enfoca o uso de Learning Analitycs sobre dados de logs de simulados realizados por alunos do ensino médio em uma plataforma virtual, buscando extrair, analisar e apresentar informações referentes a diferentes métricas de avaliação da aprendizagem, fornecendo essas informações através de relatórios e gráficos para professores e gestores com o intuito de auxiliá-los na tomada de decisão. Dentre as técnicas de LA, foram utilizadas nesse trabalho a Estatística, mais especificamente a estatística descritiva, MDE (com algoritmo de agrupamento Affinity Propagation) e Visualização da Informação (InfoVis).

Para tanto, foi utilizado o processo de Learning Analytics proposto por Chatti et al. (2012), onde os dados coletados foram pré-processados e posteriormente analisados, sendo apresentados através de análises e de informações visuais. A extração e apresentação das informações tomou como base as métricas de avaliação da aprendizagem apresentadas por Leitão et al. (2020). Para apresentação das informações foi desenvolvido um módulo Web que fornece as análises e visualizações.

Por fim, foi realizado um experimento mostrando que o uso de Learning Analytics permitiu identificar e apresentar, para professores e gestores, as turmas com menor compreensão e assertividade em relação às disciplinas avaliadas no simulado, e que nesses casos também apresentaram menores notas. Também foi possível perceber que o tempo esperado de resposta das questões, definido pelo professor, pode ter sido subestimado em algumas das disciplinas e questões, assim como algumas turmas.

\section{Trabalhos Correlatos}

Esta seção apresenta trabalhos relacionados à avaliação da aprendizagem que, em alguma medida, utilizam dados educacionais coletados em ambientes virtuais de aprendizagem para realizar a avaliação.

Nunes et al. (2016) apresentam uma proposta em que o critério avaliativo para cada aluno é baseado no cálculo de uma Nota Final que leva em consideração: (a) participação da turma, seja virtual (fóruns, mensagens, chats e frequência no AVA), seja presencial (assiduidade, resolução de exercícios em sala, cumprimento de prazos de entrega); (b) atividades executadas; e, (c) nota da prova escrita. Como feedback, são apresentados gráficos do total de alunos aprovados e reprovados por turma, relação entre a nota da participação virtual ou presencial e o número de acessos por turma.

Biswas e Ghosh (2007) apresentam um novo conjunto de métricas que considera o histórico de atividades dos estudantes, que são: (a) nível de entendimento, que leva em consideração o quão perto da resposta certa o aluno chegou, quanto tempo levou para responder e os níveis de dificuldade da questão, do tópico e do conceito abordado; (b) 
Taxa de Aprendizagem do Estudante, que indica a melhoria, na média, na pontuação de um estudante com respeito ao conjunto de avaliações.

Paiva, Bittencourt e Lemos (2019) propõem uma ferramenta de Visualização de Informação (DataViz) que busca apresentar os dados sobre os alunos de modo que possam ser facilmente compreendidos pelos professores. O trabalho apresenta 4 visualizações diferentes: (a) Gráfico de Barras Segmentado, onde as interações dos alunos são contadas e comparadas para encontrar todas as que são do mesmo tipo; (b) Pesos Ordenados, baseados no algoritmo SimpleLogistic, onde os dados são apresentados em forma de pesos; (c) Interações Combinadas, baseadas no algoritmo de regras de associação chamado JRip que apresenta quais os tipos de interação são ou não adequadas para as aulas; e, (d) Interação do curso, baseado em árvore de decisão (J48), que analisa se a aprendizagem está adequada, insuficiente ou inadequada.

Por fim, Martin e Ndoye (2016) destacam o uso de técnicas de Learning Analytics para avaliação formativa e somativa de estudantes em cursos online, de modo que utilizaram os dados de avaliação de 18 alunos em um AVA, aplicados em um período de 15 semanas. Assim, utilizaram a ferramenta Tableu, para as análises quantitativas e a ferramenta ManyEyes para a análise qualitativa. Como resultado, as análises são apresentadas para os quatro tipos de técnicas de avaliação online destacadas no trabalho: (a) avaliação do tipo de compreensão, (b) quadro de discussão, (c) focada em reflexão e (d) baseada em projeto. Também foram apresentadas diferentes formas de visualização dos dados, através de Bullet Graph, Scatter Plot e Box and Whisker Plot.

Os trabalhos de Nunes et al. (2016) e de Biswas e Ghosh (2007) apresentam diferentes métricas para avaliação da aprendizagem, que vão além da nota tradicional, porém não apresentam técnicas para análise da aprendizagem baseadas nas métricas. Já os trabalhos de Paiva, Bittencourt e Lemos (2019) e de Martin e Ndoye (2016) utilizam técnicas de Learning Analytics e InfoVis para apresentar as informações referentes aos dados de interação dos alunos com o curso e os resultados das avaliações coletados pelos ambientes virtuais, porém focam apenas nas métricas tradicionais de avaliação da aprendizagem. Neste aspecto, este trabalho utiliza, além de diferentes métricas de avaliação da aprendizagem, técnicas de Learning Analytics sobre dados de simulados fornecendo para professores e gestores informações que auxiliem na tomada de decisão.

\section{Método de Extração das Informações}

Esta seção apresenta o conjunto de dados e métricas utilizados neste trabalho, assim como o método de extração, análise e apresentação das informações.

\subsection{Métricas de Avaliação da Aprendizagem}

Para geração das informações, este trabalho utiliza além da Nota Tradicional, que é relativa ao número de questões corretas, 7 diferentes métricas de avaliação da aprendizagem apresentadas em Leitão et. al. (2020), sendo: (a) Nota Ponderada, que considera um peso para cada alternativa de resposta conforme a proximidade da alternativa em relação a resposta correta; (b) Tempo de Resposta, que é o tempo que o aluno precisou para resolver cada questão; (c) Tempo Esperado, é o tempo estimado pelo professor para resolução da questão; (d) Nível de Compreensão, que busca estimar a compreensão do aluno em relação a questão ou ao questionário, levando em consideração a dificuldade da questão, dificuldade do conteúdo, o peso da alternativa 
marcada e o Tempo de Resposta; (e) Dúvida, quantifica o número de vezes que o aluno alterou sua resposta em cada questão; (f) Assertividade, que mede a autoconfiança do aluno com base no número de questões corretas e a soma do número de vezes que o aluno alterou suas respostas; e (g) Prioridade, que define valores de prioridade para as disciplinas e tópicos baseada na diferença entre a Nota Tradicional e Nota Ponderada.

\subsection{Extração das Informações}

Para a extração das informações e geração das análises foram seguidas as fases do processo de Learning Analytics proposta por Chatti et al. (2012). Na fase de Coleta e Pré-Processamento, foi alimentado um banco de dados não relacional, o MongoDB, com os dados de interação dos alunos ao responderem aos simulados na Plataforma SIGNAL, desenvolvida por Leitão (2017), e a partir desses dados foram extraídas as informações e calculados os valores das métricas.

A fase de Análise e Ação contou com técnicas de estatística descritiva e mineração de dados educacionais para extrair informações úteis da base de dados. Como o objetivo da aplicação de Learning Analytics nesse trabalho foi o monitoramento e análise, conforme os objetivos definidos em Chatti et al. (2012), buscou-se apresentar informações que auxiliem professores e gestores na tomada de decisão.

$\mathrm{Na}$ fase de Pós-Processamento, as informações geradas na fase anterior foram gravadas em dataframes, permitindo que a partir das análises e informações iniciais os professores e gestores possam personalizar a visualização das informações através da filtragem e combinação das mesmas, obtendo assim novas análises.

\subsection{Módulo de Análise}

A partir da extração das informações, foi alimentado um módulo web (Figura 1) que automatiza e disponibiliza análises e gráficos interativos para os professores e gestores utilizando as técnicas de Learning Analytics, permitindo assim que os tomadores de decisões obtenham informações referentes a aprendizagem dos alunos.

O módulo de análise foi criado utilizando a linguagem Python, com bibliotecas voltadas para mineração e análise de dados como Pandas, Scikit-learn e a ferramenta Plotly para geração de gráficos interativos. O módulo possui uma área de Controle e uma de Análise/Informação, sendo que na área de Controle é possível selecionar a turma, disciplina e tópico que se deseja analisar. A área de Informação possui diferentes abas, com explicações sobre o módulo e as métricas utilizadas, assim como a apresentação das análises e visualizações de informações geradas sobre essas turmas, disciplinas, tópicos e questões avaliadas.

A partir do uso de Learning Analytics, principalmente de estatística descritiva, foram geradas análises referentes a todas as turmas, ou nos casos em que uma turma é selecionada as informações apresentadas são referentes aquela turma. As análises das turmas incluem os valores das métricas, percentual de reprovação da turma, assim como as disciplinas em que os alunos obtiveram maior e menor Nível de Compreensão, e as que receberam maior valor na métrica Prioridade, buscando assim indicar para professores e gestores as disciplinas em que os alunos necessitam de maior atenção. 

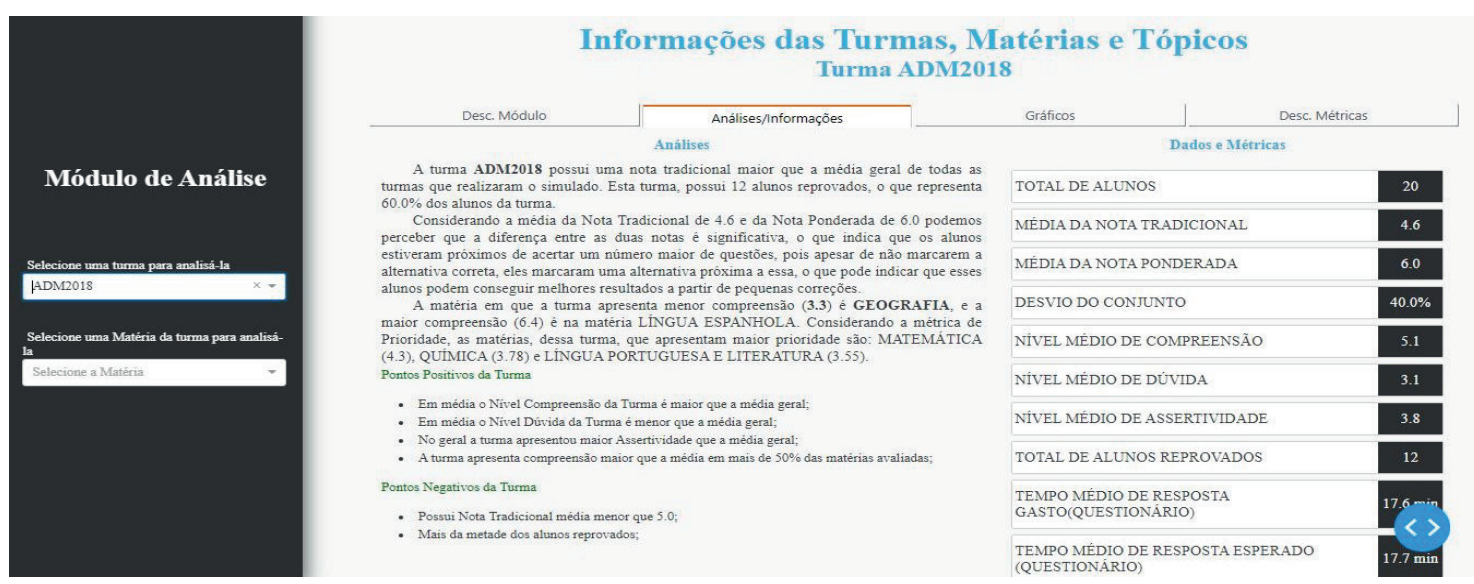

Figura 1: Visão geral do Módulo de Análise desenvolvido.

Dentre as análises por turma, são destacados pontos positivos e negativos de cada uma, onde são comparados os valores das métricas obtidas pela turma com os valores médios de todas as turmas. Além disso, também é informado se a turma obteve um Nível de Compreensão acima ou abaixo da média geral em mais da metade das disciplinas avaliadas. Essa informação é importante pois permite verificar se os alunos apresentam baixo Nível de Compreensão em disciplinas pontuais ou se na maioria.

\section{Experimento e Discussões}

Com o intuito de avaliar as informações geradas pela ferramenta com o uso de Learning Analytics, foi executado um experimento com os dados de simulados de alunos do ensino médio técnico. Nesse experimento foram coletados dados de simulados aplicados através de um AVA para alunos de 11 turmas do ensino médio integrado (cursos técnicos em Administração, Informática e Recursos Pesqueiros) entre os anos de 2017 e 2019.

Tabela 1: Dados dos simulados na base de dados do SIGNAL.

\begin{tabular}{|c|c|c|c|}
\hline Turma & $\begin{array}{c}\mathbf{N}^{\mathbf{0}} \text { de Alunos } \\
\text { avaliados }\end{array}$ & $\begin{array}{c}\mathbf{N}^{\mathbf{0}} \text { de questionários no } \\
\text { simulado }\end{array}$ & $\begin{array}{c}\text { Questões por } \\
\text { simulado }\end{array}$ \\
\hline $\mathbf{1}$ & 33 & 9 & 40 \\
\hline $\mathbf{2}$ & 28 & 9 & 44 \\
\hline $\mathbf{3}$ & 29 & 9 & 44 \\
\hline $\mathbf{4}$ & 16 & 9 & 40 \\
\hline $\mathbf{5}$ & 34 & 9 & 40 \\
\hline $\mathbf{6}$ & 20 & 8 & 50 \\
\hline $\mathbf{7}$ & 27 & 5 & 35 \\
\hline $\mathbf{8}$ & 24 & 8 & 50 \\
\hline $\mathbf{9}$ & 18 & 5 & 35 \\
\hline $\mathbf{1 0}$ & 20 & 8 & 50 \\
\hline $\mathbf{1 1}$ & 39 & 8 & 50 \\
\hline
\end{tabular}

Cada simulado possui diferentes questionários, e cada questão do questionário apresenta 5 opções de resposta. Os simulados foram aplicados a alunos de $1^{\circ}, 2^{\circ}$ e $3^{\circ}$ ano e deveriam ser respondidos no tempo máximo de 4 horas. Até o momento, cada turma 
participou de apenas um simulado, conforme é detalhado na Erro! Fonte de referência não encontrada..

Atualmente, a base de dados dos simulados conta com dados de 8 simulados diferentes, cada um com 5 a 9 questionários, compostos por 35 a 50 questões. Cada questionário pertence a uma disciplina específica, sendo elas: Língua Portuguesa e Literatura, Língua Inglesa, Língua Espanhola, História, Química, Física, Sociologia, Matemática, Geografia, Educação Física e Biologia.

Do total de 339 alunos registrados, apenas 295 participaram dos simulados, porém durante o pré-processamento dos dados foram removidos os outliers relacionados a métrica de dúvida utilizando o método z-score, restando assim um total de 288 estudantes analisados no experimento.

\subsection{Análises e Visualizações}

Considerando a média da Nota Tradicional dos alunos de todas as turmas, e que a nota mínima para aprovação no simulado é de 5.0, a ferramenta mostrou que $77,8 \%$ dos alunos foram reprovados contra apenas $22,2 \%$ de aprovados. A Figura 2 apresenta $o$ percentual de alunos aprovados e reprovados em cada turma, considerando tanto a Nota Tradicional dos alunos (barras azuis e vermelhas), quanto a Nota Ponderada (barras verdes e lilás), permitindo que se visualize essa informação mais rapidamente.

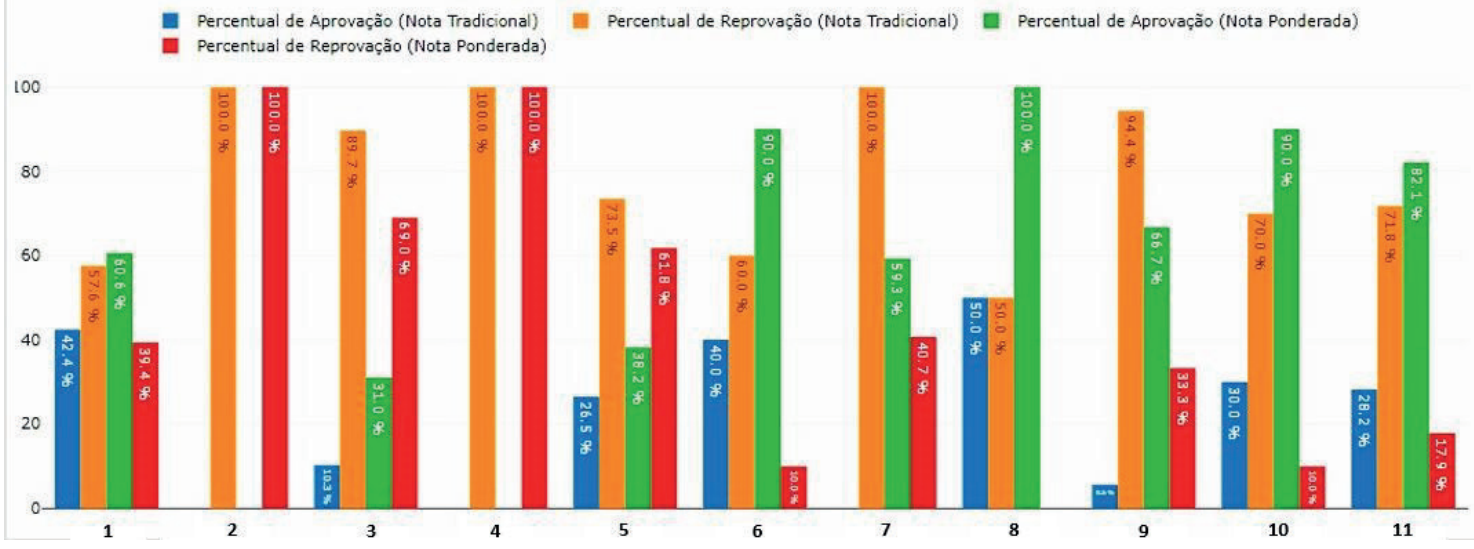

Figura 2: Percentual de aprovação e reprovação nas turmas.

O gráfico da Figura 2 mostra que, ao considerarmos apenas as Notas Tradicionais, o percentual de aprovação (barras azuis) tende a ficar abaixo de $50 \% \mathrm{em}$ quase todas as turmas, sendo que o maior percentual de aprovação é de 50\% (Turma 8). Entretanto, ao considerarmos as Notas Ponderadas dos alunos esse percentual tende a subir, chegando a $100 \%$ de aprovação na turma 8 . Essa informação permite que os tomadores de decisão visualizem quais as turmas que, apesar da Nota Tradicional mais baixa, estiveram próximas de acertarem um maior número de questões, pois, uma Nota Ponderada mais elevada significa que foram marcadas alternativas com pesos maiores.

A Figura 2 também nos mostra que as turmas 2 e 4, mantiveram percentual de reprovação idêntico (100\%) quando analisadas ambas as notas, o que demostra um cenário preocupante em virtude do baixo desempenho alcançado pelas referidas turmas. Quando analisamos os valores das demais métricas percebemos que a Turma 2 apresentou um valor de Dúvida elevado, ao mesmo tempo que apresentou baixas Assertividade e Nível de Compreensão. A combinação dessas informações busca 
mostrar quais as turmas em que os alunos apresentam menor domínio do conteúdo avaliado, nesse caso, destacamos novamente a turma 2 .

Já o gráfico da Figura 3, traz a diferença entre o Tempo de Resposta médio e a média do Tempo Esperado de cada questão, usando minutos como unidade de medida. Como podemos visualizar na Figura 3, quase todas as turmas apresentaram um tempo gasto maior que o esperado, o que auxilia na compreensão de que o tempo estipulado para resolução da questão foi subestimado pelo professor, pois os alunos demandaram mais tempo, permitindo que os professores possam decidir por revisar esse tempo.

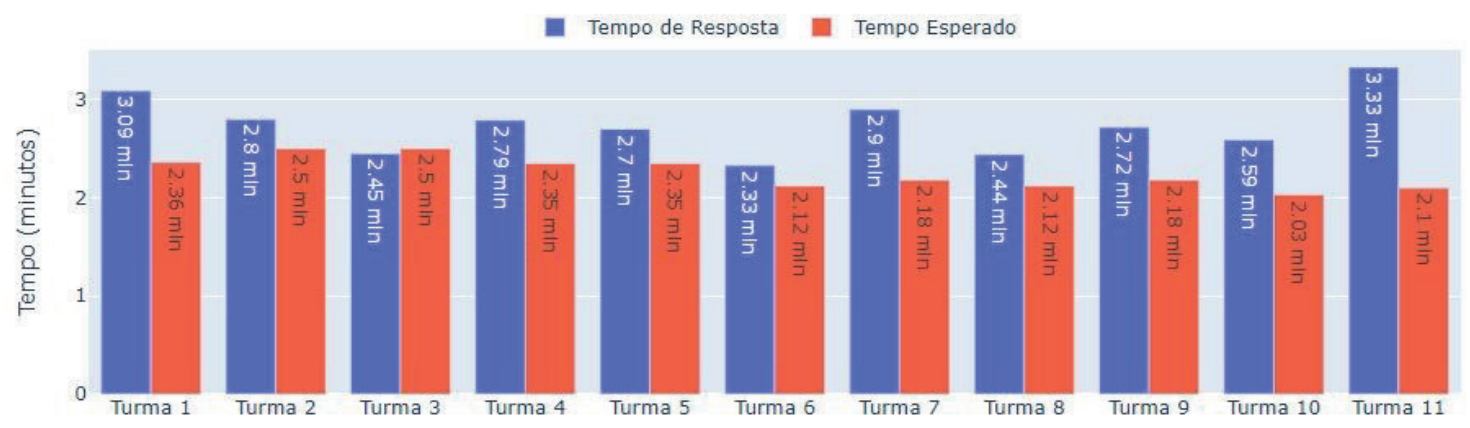

Figura 3: Comparação entre o Tempo de Resposta gasto pelos alunos e o Tempo de resposta esperado pelo professor.

Uma análise do tempo de resposta por disciplina mostrou que as disciplinas de Biologia, Língua Inglesa, Matemática e Química apresentaram em média o Tempo de Resposta maior que Tempo Esperado, o que permite aos professores avaliar se há a necessidade de mais tempo para interpretação da questão ou para realização de cálculos.

Em contrapartida, a disciplina de Língua Espanhola e Geografia apresentaram a média do Tempo de Resposta por questão menor que o tempo estipulado pelo professor, o que poderia indicar que o professor superestimou o tempo necessário ou que os alunos marcaram a alternativa sem avaliar a questão, ou seja, "chutaram" a resposta. A informação sobre a quantidade de alunos que marcaram a alternativa em um tempo muito menor que o estipulado também é fornecida por questão.

Outra informação importante que o uso de Learning Analytics apresentou foi o desempenho dos alunos de cada turma em relação as disciplinas avaliadas no simulado. A Figura 4 mostra, através de um mapa de calor, o Nível de Compreensão de cada turma (eixo x) em relação as disciplinas (eixo y), sendo que as cores mais claras representam maior compreensão, e as mais escuras representam menor compreensão. Nos casos em que há valores ausentes significa que o simulado realizado por aquela turma não incluiu a referida disciplina.

O gráfico da Figura 4 traz um conjunto de informações relevantes, pois permite visualizar quais disciplinas necessitam de intervenções pontuais em algumas turmas e quais as disciplinas que apresentam menor ou maior compreensão na maioria das turmas. Através do mapa de calor, observa-se que as disciplinas de Geografia e Educação Física apresentam valores de compreensão mais baixos (cores mais escuras) em quase todas as turmas enquanto as disciplinas de História, Língua Inglesa, L. Portuguesa e Literatura e Química apresentaram melhores Níveis de Compreensão.

Ao mesmo tempo que permite analisar as disciplinas, a Figura 4 também mostra que a Turma 2 apresentou valores baixos de compreensão (considerando a média geral 
que é de 4,6) na maioria das disciplinas, especialmente em Língua Espanhola e Geografia, seguida pela Turma 4 que também apresentou baixa compreensão em diversas disciplinas, principalmente em Educação Física.

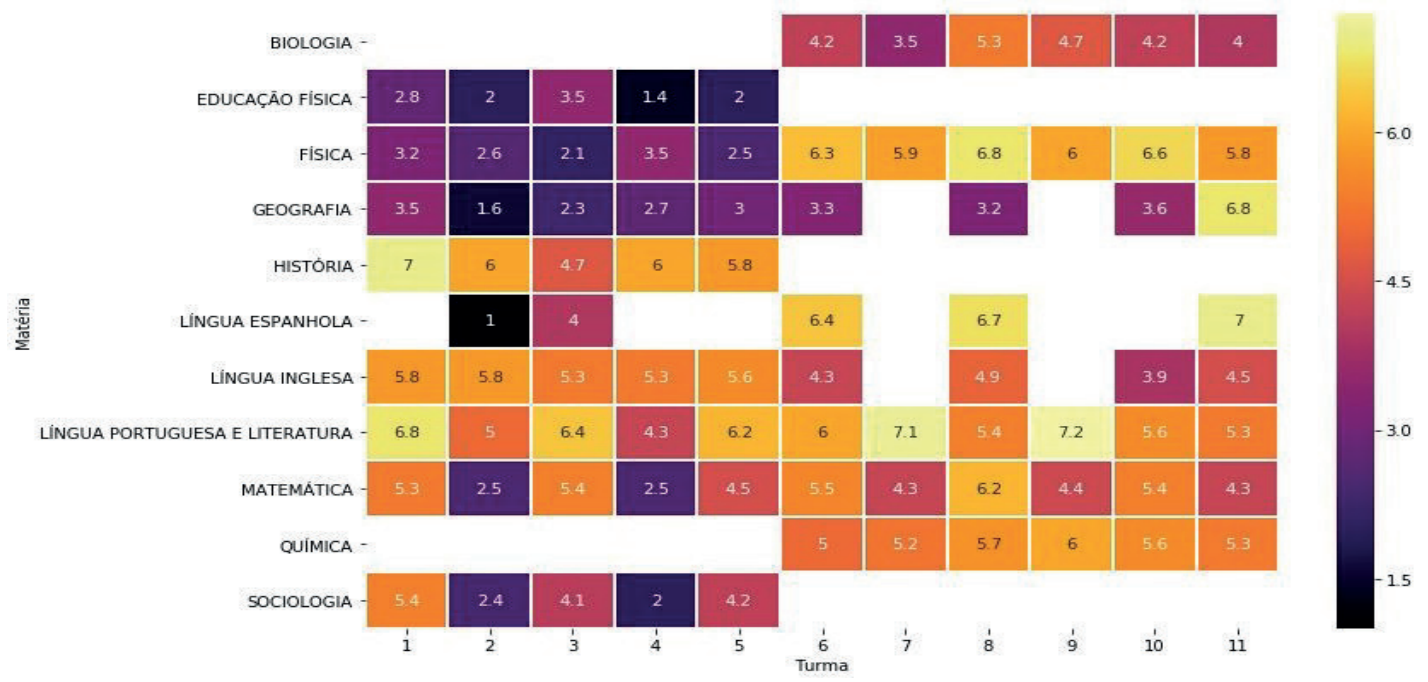

Figura 4: Nível de Compreensão das turmas por disciplina.

Com exceção das disciplinas que apresentam valores baixos de compreensão na maioria das turmas, algumas precisam de intervenção em turmas específicas, como é o caso de Língua Inglesa e Geografia na Turma 10, ou Biologia e Matemática na Turma 7. Em resumo, o mapa de calor indica para professores e gestores quais disciplinas necessitam de mais atenção em cada turma, pois a mesma disciplina pode ter um valor de compreensão diferente para cada uma, permitindo assim que sejam tomadas decisões personalizadas em cada turma.

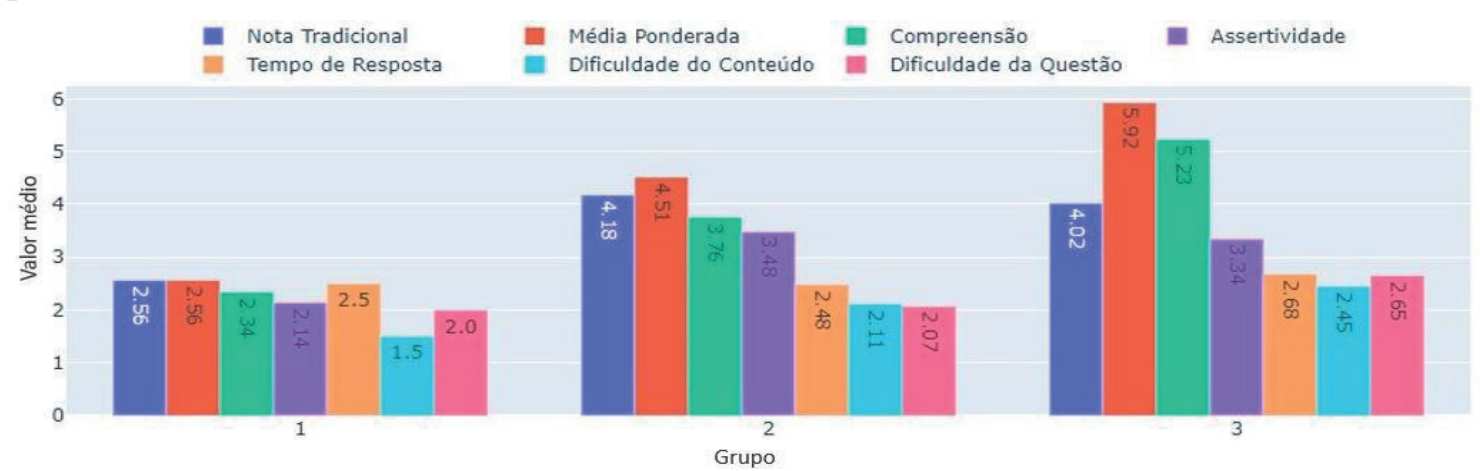

Figura 5: Valores médios das métricas por grupo.

Buscando encontrar novas informações, foi utilizado o algoritmo Affinity Propagation para agrupamento das disciplinas baseado nos valores das métricas de avaliação da aprendizagem, assim como valores de Dificuldade do Conteúdo e Dificuldade da Questão, que foram definidos pelo professor. Ao final da execução, o algoritmo gerou 3 grupos de disciplinas, onde o grupo 1 conta com Educação Física, enquanto o grupo 2 possui Biologia, Geografia e Sociologia, e o grupo 3 possui Física, História, L. Espanhola, L. Inglesa, L. Portuguesa e Literatura, Matemática e Química. Os valores médios das métricas em cada grupo podem ser visualizados na Figura 5. 
O gráfico apresentado na Figura 5 permite perceber que as disciplinas do grupo 3 apresentam melhor Nota Ponderada e Nível de Compreensão, mesmo com maiores dificuldades dos conteúdos e das questões, enquanto o grupo 2 possui menor dificuldade de conteúdo e questões, porém apresenta Nota Tradicional e Assertividade maiores. Esse agrupamento também pode servir para que os professores verifiquem quais as disciplinas estão no grupo com menor desempenho, independente dos valores de dificuldade, facilitando o processo de intervenção.

As informações apresentadas pela ferramenta se encaixam em alguns dos objetivos do processo de avaliação sumarizados por Biswas e Ghosh (2007), como: reunir informações sobre características dos alunos que permitam definir o progresso (ou falta dele) e ações corretivas que precisam ser tomadas, fornecer informações a um professor que o auxilie a julgar a eficácia dos métodos de ensino, entre outros aspectos.

\section{Conclusão}

A contribuição principal dessa pesquisa é fornecer para professores e gestores diferentes e relevantes informações referentes à avaliação da aprendizagem dos alunos a partir da aplicação de Learning Analytics sobre os dados de simulados. Essas informações e análises poderão auxiliar na compreensão dos resultados alcançados pelos estudantes e na tomada de decisões.

As análises e visualizações da ferramenta fornecem novas informações sobre a aprendizagem e o desempenho dos estudantes em cada disciplina e em cada turma, sendo que uma análise mais detalhada é fornecida pois em muitos casos a mesma disciplina pode ser ministrada em turmas diferentes e por professores diferentes, permitindo que os professores possam compartilhar informações e casos de sucesso, baseados nos dados de compreensão e demais métricas apresentadas.

A partir da verificação do Tempo de Resposta e das demais métricas, há indícios de que os alunos tenderam a marcar alternativas sem avaliar a questão (marcação cega ou "chute"), o que pode evidenciar uma falta de compreensão sobre o assunto avaliado, a pergunta da questão ou a disciplina, por exemplo. Uma análise mais extensa será realizada com as turmas 2 e 4 pois as turmas apresentam baixa compreensão na maioria das disciplinas e para tentar entender melhor o percentual de $100 \%$ de reprovação, mesmo considerando a nota ponderada.

Em resumo, o experimento com a ferramenta desenvolvida mostrou que o uso de Learning Analytics sobre dados de simulados podem trazer resultados importantes para o processo de avaliação da aprendizagem e desempenho, auxiliando assim os tomadores de decisão a obter informações sobre os alunos, que vão além da Nota Tradicional.

Como trabalhos futuros, pretende-se adicionar novas análises a partir do uso de Learning Analytics, assim como utilizar novas métricas de avaliação da aprendizagem. Outros experimentos poderão ser realizados com outras turmas a partir dos dados de novos simulados. O sistema desenvolvido será validado junto aos professores e gestores, podendo ser melhorado a partir do resultado da validação. 


\section{Agradecimentos}

O presente trabalho foi realizado com apoio da Coordenação de Aperfeiçoamento de Pessoal de Nivel Superior - Brasil (CAPES) - Código de Financiamento 001. Esta pesquisa, conforme previsto no Art. 48 do decreto $n^{\circ} 6.008 / 2006$, foi parcialmente financiada pela Samsung Eletrônica da Amazônia Ltda, nos termos da Lei Federal $n^{o}$ 8.387/1991, através do convênio $n^{\circ}$ 003/2019, firmado com o ICOMP/UFAM. Os autores também agradecem o apoio financeiro parcial da Fundação de Amparo à Pesquisa do Estado do Amazonas através do Projeto 122/2018 (UNIVERSAL).

\section{Referências}

Biswas P. e Ghosh S. A Novel Approach to Define Performance Metrics for Students' and Teachers' Evaluation. Electronic Journal of e-Learning, 5(2):87-102, 2007.

Chatti, M. A.; Dyckhoff, A. L.; Schroeder, U.; Thüs, H. A reference model for learning analytics. International Journal of Technology Enhanced Learning, v. 4, n. 5-6, p. 318-331, 2012.

Filvá, D. A.; Alier, M.; Casany, M. J.; Mayo, E. A learning analytics tool with hybrid graphical and textual interpretation generation. ACM International Conference Proceeding Series, v. 02-04-Nove, p. 326-333, 2016.

Frey, B. J.; Dueck, D. Response to comment on "clustering by passing messages between data points. Science, v. 315, p. 972-977, fev. 2007.

Leitão, G. D. S. Uma Plataforma de Suporte ao Docente no Contexto da Educação Digital. Manaus/AM: UFAM, 2017. 93p. Dissertação de Mestrado.

Leitão, G.; Colonna, J; Monteiro, E.; Oliveira, E.; Barreto, Raimundo. New Metrics for Learning Evaluation in Digital Education Platforms. 2020. Disponível em: $<$ https://arxiv.org/abs/2006.14711>. Acesso em: 01 nov 2020.

Martic, M. Evaluation of quiz using a statistical calculation in Learning Management System. In: 25th International Conference on Software, Telecommunications and Computer Networks (SoftCOM), v. 0, p. 5, 2017.

Martin, F., Ndoye, A. Using learning analytics to assess student learning in online courses. Journal of University Teaching and Learning Practice, v. 13, 2016.

Nunes, I., Silva, W.M., Laisa, J., Ugulino, F. e Lucena, M., Learning Analytics como ferramenta para a análise do desempenho dos alunos em Cursos Semipresenciais. In: XVII Simpósio Brasileiro de Informática na Educação (SBIE). Anais. Congresso Brasileiro de Informática na Educação - CBIE, 2016.

Paiva, R., Bittencourt, I. I. e Lemos, W. Helping Teachers Visualize Students' Performance. In: XXX Simpósio Brasileiro de Informática na Educação (SBIE), p. 1731-1740, 2019, Brasília/DF. Anais. CBIE, 2019.

Pineda, A. F.; Cadavid, J. M. A systematic literature review in Learning Analytics. In: Workshop de Ciência de Dados Educacionais (WCDE), Anais.CBIE, p. 429,2018.

Siemens, G. Learning Analytics \& Knowledge: February 27-March 1. $1^{\text {st }}$ International Conference on Learning Analytics and Knowledge 2011. Banff, Alberta. 2010. Disponível em: <https://tekri.athabascau.ca/analytics/>. Acesso em: 01 nov. 2020. 